\title{
The management of interstitial cystitis: an update
}

\author{
Sagar Phatak and Harris E Foster Jr ${ }^{*}$
}

\section{SUMMARY}

Treating interstitial cystitis (IC) is one of the greatest challenges facing physicians and other health care providers who manage patients with this condition. The symptoms of urinary frequency and urgency, dysuria, and chronic pelvic pain characterize IC, but it is the debilitating pelvic pain associated with IC that is most difficult to control. The pathophysiology of IC pain is poorly understood, but is thought to be a complex entity including nociceptive, visceral, and neuropathic components. There are currently no universally effective therapies available. Oral treatments, however, are frequently used, including nonsteroidal anti-inflammatory drugs, tricyclic antidepressants, gabapentin, and pentosan polysulfate, all of which have shown varying degrees of efficacy. Recognition that IC pain is multifactorial, and probably has a neuropathic component, has led to the use of some of these agents, previously prescribed for other neurologic conditions associated with chronic pain. Intravesical and surgical options are also available, which expands the armamentarium for those who treat pain secondary to IC. Treating IC requires managing all of the symptoms of this disease. This review aims to cover standard and novel treatment options, while concentrating on the management of pain.

\section{KEYWORDS chronic pelvic pain, interstitial cystitis, neuropathic pain}

\section{REVIEW CRITERIA}

Information presented in this article was obtained by a review of the literature on pain management for interstitial cystitis using both the PubMed and Ovid medical databases for articles published between January 1980 and August 2005 using the keywords "interstitial cystitis", "chronic pelvic pain", "clinical trials", and "bladder physiology".
$S$ Phatak is a Resident in Urology and HE Foster Jr is an Associate Professor of Surgery in the Section of Urology, Yale University School of Medicine, New Haven, CT, USA.

\section{Correspondence}

*Department of Surgery, Section of Urology, Yale University School of Medicine, PO Box 208041, New Haven, CT 06520-8041, USA

harris.foster@yale.edu

Received 2 August 2005 Accepted 21 November 2005

www.nature.com/clinicalpractice

doi:10.1038/ncpuro0385

\section{INTRODUCTION}

Interstitial cystitis (IC) treatment primarily involves managing patients' symptoms in order to improve their quality of life. This difficult-totreat disease, with an etiology as yet undefined, is a benign disorder that, nonetheless, can have devastating effects on a patient's physical and mental health. Although urinary frequency and urgency (both symptoms of overactive bladder) are problematic for the patient, pain remains the hallmark of this disease and can be difficult to manage, which is frustrating to both the patient and the treating physician. This review covers the current pharmacologic and nonpharmacologic management options for IC, with an emphasis on pain management. The relative efficacy of different treatments for IC will be reviewed, including nonsteroidal anti-inflammatory drugs (NSAIDs), gabapentin, pentosan polysulfate, antidepressants, intravesical agents, and surgery.

\section{ETIOLOGY OF INTERSTITIAL CYSTITIS}

IC is a chronic, noninfectious, probably inflammatory disorder of the urinary bladder that primarily affects women (approximately $90 \%$ of patients are female). ${ }^{1}$ The disease can also affect men and possibly children; however, these groups of patients have been studied or characterized to a lesser extent than women. It is believed that IC prevalence ranges from 1 in 100,000 to 5.1 in 1,000 of the general population worldwide, and by definition the symptoms include some component of pain, usually CHRONIC PELVIC PAIN. ${ }^{2,3}$ Some authors think that the diagnosis of NONBACTERIAL CHRONIC PROSTATITIS in men might actually represent IC. ${ }^{4}$ Symptoms of IC include urinary frequency and urgency, dyspareunia, and, most commonly, debilitating pelvic pain. It is believed that symptoms might be progressive if left untreated, and can severely decrease patients' quality of life. The symptoms of IC can lead to depression, social isolation, and, in rare instances, suicidal thoughts. ${ }^{5}$

The pathophysiology of IC remains elusive; many theories have been formulated, and in part 


\section{GLOSSARY}

CHRONIC PELVIC PAIN

Nonmenstrual pain of

3 months duration or

longer that localizes to

the anatomic pelvis and is

severe enough to cause

functional disability

NONBACTERIAL

CHRONIC PROSTATITIS

A condition affecting

patients who present with

symptoms of prostatitis

without a positive urine

culture or expressed

prostate secretion culture;

symptoms often include

pelvic pain, penile and

perineal pain, dysuria

HUNNER'S ULCER

Characteristic mucosal ulcer seen in classic, ulcerative interstitial cystitis (in 10\% of patients with interstitial cystitis)

\section{GLOMERULATIONS}

Objective finding in the diagnosis of interstitial cystitis characterized by petechial hemorrhages that occur after distension of the urinary bladder have driven therapy. The bladder transitionalcell epithelium is normally covered by a mucin layer composed of glycosaminoglycans. This layer is thought to be almost impermeable, thereby preventing urine solutes from diffusing into the subepithelial components of the bladder. IC might affect this layer by increasing solute permeability, possibly leading to irritation, inflammation, and sensory-nerve sensitization of the bladder. ${ }^{5}$ Potassium could be the main offending substance, and its diffusion across the permeable transitional epithelium the primary irritant; hence the development of the potassium sensitivity test for the diagnosis of IC.

\section{DIAGNOSIS AND MANAGEMENT OF INTERSTITIAL CYSTITIS}

Traditionally, diagnosis of IC includes obtaining a complete patient history and conducting a physical examination. Cystoscopy with hydrodistension under anesthesia is often performed, in an attempt to detect the characteristic features of a HUNNER'S ULCER and/or GLOMERULATIONS. The introduction of the potassium sensitivity test and questionnaires such as the O'Leary-Sant Interstitial Cystitis Symptom Index and the Pelvic Pain, Urgency and Frequency Questionnaire have provided further tools to assist in the diagnosis of IC. ${ }^{6,7}$ It is critical, however, that a thorough evaluation first rules out any reversible causes of patient symptoms, including infection, cancer, urolithiasis, obstruction, neurologic disease, or gynecologic disorders such as endometriosis. Once other causes are excluded and a diagnosis of IC is confirmed, therapy should be aimed at controlling symptoms, particularly pelvic pain, which can often be debilitating.

Pain can be classified as nociceptive, visceral, or neuropathic. IC pain is rather complex and can often include all three types of pain, making management extremely difficult. The most successful approaches have been multimodal therapies that incorporate psychologic interventions, physical therapy, pharmacotherapy and in some cases procedural treatments, such as sacral neuromodulation. Attempting to address all aspects of pain management is beyond the scope of this review. This paper will specifically outline the current pharmacologic options available for managing IC pain, including some new developments.

It should be noted that there are few wellperformed, placebo-controlled, randomized trials that have investigated the various IC treatments. Historically, for a variety of reasons (i.e. chronic pain syndrome, low incidence of disease), it has been difficult to enroll IC patients in randomized studies, particularly those that involve the use of a placebo. As there are no universally successful therapies, randomized clinical trials for IC should include a placebo arm whenever possible. It has been suggested that limiting the length of treatment and follow-up, allowing open-label treatment at completion, and/or providing appropriate, reasonable compensation can partly ameliorate the potential problems experienced by patients in the placebo arm. ${ }^{8}$

\section{ORAL TREATMENTS FOR INTERSTITIAL CYSTITIS \\ Nonsteroidal anti-inflammatory drugs, narcotics, and antihistamines}

The initial management of most chronic pain conditions including IC should involve NSAIDs, and, if needed, narcotics. Unfortunately, NSAIDs and narcotics have been ineffective in many patients with IC. ${ }^{9,10}$ The anti-inflammatory effects of NSAIDs might help to reduce some bladder inflammation (assuming it exists), thereby reducing pain. Specifically, these agents might relieve symptoms in a minority of patients by the inhibition of prostaglandin formation.

It is believed that narcotics affect Type $\mathrm{C}$ postganglionic pain fibers that possess slow conduction. These pain fibers are characterized by a dull, aching, 'deep' pain. ${ }^{11}$ Extended-release formulations of oxycodone and morphine are likely to provide better pain relief than short-acting agents such as meperidine. Narcotics should be introduced in gradually increasing doses, starting with small doses at set time intervals, allowing for 'rescue doses' if patients experience recurrence of pain. In addition to oral agents, some drugs (such as lidocaine) can be given intravesically as a $1-2 \%$ solution for acute flare episodes in IC. ${ }^{12}$ Improvement in pain scores when lidocaine is combined with intravesical heparin occurs in $50 \%$ to $94 \%$ of patients. ${ }^{13}$ There might also be some potential efficacy with lidocaine when delivered via a transdermal patch, although further study is needed.

Nalmefene is a long-acting opiate antagonist that has been used to treat IC pain with only limited success. The drug was originally developed as a long-acting version of naloxone, with the primary function of reversing the depression of the respiratory and central 
Table 1 Summary of oral therapy for interstitial cystitis.

\begin{tabular}{|c|c|c|}
\hline Therapy & Efficacy of treatment & Evidence of efficacy \\
\hline $\begin{array}{l}\text { Nonsteroidal anti-inflammatory } \\
\text { drugs and narcotics }\end{array}$ & Variable & Open-label trials ${ }^{9-13}$ \\
\hline Nalmefene & $\begin{array}{l}50 \% \text { of patients reported } \\
\text { improvements in pain symptoms }\end{array}$ & Phase II trial ${ }^{15}$ \\
\hline Hydroxyzine & No benefit over placebo & Randomized, controlled trial ${ }^{18}$ \\
\hline Pentosan polysulfate & $\begin{array}{l}45-50 \% \text { of patients responded by } \\
32 \text { weeks of treatment }\end{array}$ & Randomized, controlled trial ${ }^{24}$ \\
\hline Amitriptyline & $\begin{array}{l}63 \% \text { of patients were satisfied with } \\
\text { treatment }\end{array}$ & Randomized, controlled trial ${ }^{26}$ \\
\hline Gabapentin & $\begin{array}{l}50 \% \text { of patients reported } \\
\text { improvements in pain symptoms }\end{array}$ & Case series ${ }^{31}$ \\
\hline Prednisone & $\begin{array}{l}69 \% \text { of patients reported } \\
\text { improvements in pain symptoms }\end{array}$ & Case series and open-label trials ${ }^{35}$ \\
\hline Cyclosporine ${ }^{a}$ & $\begin{array}{l}90 \% \text { of patients reported no bladder } \\
\text { pain }\end{array}$ & Open-label tria| ${ }^{37}$ \\
\hline
\end{tabular}

nervous systems observed in opioid intoxication. ${ }^{14}$ It was, however, observed to decrease mast-cell degranulation, and as such was tested in a phase II clinical trial to treat IC. ${ }^{15}$ That study in 1994 did show a 50\% decrease in suprapubic bladder pain; however, no other studies have been published on the efficacy of this drug in the treatment of IC pain. ${ }^{15}$ As such, while nalmefene is available, its use in the treatment of IC requires further study.

Antihistamines have also been used to treat IC in open-label studies. The number of mast cells has been shown to be increased in bladder biopsies from some IC patients. ${ }^{16}$ Decreasing mast-cell activity might, therefore, decrease bladder inflammation. As histamine, released in many instances by mast cells, has been implicated in the pathophysiology of IC, antihistamines have been used with varying efficacy for the treatment of this disease. The most commonly used antihistamine for IC has been hydroxyzine, ${ }^{17-19}$ which acts by blocking both mast-cell secretion and the $\mathrm{H}_{1}$ receptor. An additional neurogenic mechanism of mast-cell release involves SUBSTANCE $\mathrm{P} ;{ }^{20}$ Studies in animal models have demonstrated that the release of substance $P$ is inhibited by hydroxyzine. ${ }^{21}$ Inhibition of histamine and substance $\mathrm{P}$ release are thought to lessen the ensuing hyperemia that is associated with both of these substances, and reduce the fibrosis associated with substance $\mathrm{P}$.

In an open-label study, the use of hydroxyzine was shown to improve symptom scores by $40 \%$ from baseline. ${ }^{17}$ Drowsiness, however, is a common adverse effect associated with antihistamines, but might abate after chronic use. A randomized trial comparing hydroxyzine to pentosan polysulfate either alone or in combination, however, failed to show a significant advantage over placebo. ${ }^{18}$ Other promising areas of research have been aimed at regulating mast-cell activity. Degranulation of mast cells can occur when their opiate receptors are stimulated, and inhibition of these receptors by an opiate antagonist such as nalmefene has demonstrated some efficacy. ${ }^{15,19}$

In summary, while the classes of oral agents discussed above might help some patients, many will often require additional agents to ameliorate their IC symptoms, particularly the pain. The most effective treatment will include concomitant therapy with additional oral agents such as pentosan polysulfate, antidepressants, or gabapentin (Table 1$){ }^{22}$

\section{Pentosan polysulfate}

An oral agent frequently used to treat the symptoms of IC is pentosan polysulfate sodium (PPS). PPS remains the only FDA-approved oral agent for the treatment of IC. An oral heparinoid, it is thought to augment the glycosaminoglycan layer of the bladder; repairing deficiencies in this layer might lead to improvement of IC symptoms. The use of PPS for IC was originally described in 1990 and continues to be investigated. ${ }^{23}$

A randomized, controlled trial investigating the use of PPS to treat IC that was published in 2005 is the most vigorous study to date. ${ }^{24}$ Various

\section{GLOSSARY}

SUBSTANCE P

11-aminoacid-neuropeptide that functions in the transmission of pain impulses from peripheral receptors to the central nervous system 
www.nature.com/clinicalpractice/uro

\section{GLOSSARY}

5-HYDROXYTRYPTAMINE

A neurotransmitter

associated with the sleep

cycle, which is believed to

play a role in depression,

bipolar disorder, and anxiety doses have been tested (ranging from $300 \mathrm{mg}$ to $900 \mathrm{mg}$ once a day), but overall response rates approach $15-67 \%$ at the $300 \mathrm{mg}$ dose, with no significant improvement seen with higher doses. ${ }^{24}$ Ultimately, the duration of dosage appears to be more important than the level of dosage itself. The severity of symptoms does not appear to affect patients' responses to pentosan polysulfate. Modest improvements in symptoms have been described at 4 weeks into therapy, with continued steady improvements over time. ${ }^{24}$

It is often difficult to delineate symptoms within IC, and determining response to treatment is aimed at evaluating improvement of IC as an entity, rather than just the analgesic effects. On closer inspection of the 2005 study, however, this agent seems to improve urinary frequency symptoms more than pain. ${ }^{24}$ Adverse effects associated with PPS have generally been modest and resolve without intervention or long-term sequelae. The most common treatment-related adverse effects of PPS include gastrointestinal disorders, headache, and alopecia. ${ }^{24}$

\section{Tricyclic antidepressants}

Many of the agents previously discussed are aimed at both the nociceptive and visceral pain associated with IC. The tricyclic antidepressant amitriptyline, however, is directed at treating the presumed neuropathic pain component of IC. In 1987, Hanno and Wein first described the use of amitriptyline in the treatment of IC. ${ }^{25}$ Its anecdotal efficacy has led to it becoming one of the most frequently prescribed oral agents for IC. ${ }^{9}$ It was not, however, until 2004 that amitriptyline was shown to be effective in a randomized, placebo-controlled, double-blind trial. In this report, $63 \%$ of patients reported satisfaction with therapy compared to $4 \%$ in the placebo group. ${ }^{26}$ Specifically, mean pain intensity, as measured by a visual analog scale, revealed statistically significant improvements in the amitriptyline group, but not in those who received placebo. The mechanism of pain relief obtained with amitriptyline treatment is still not completely understood. Its effects on urinary frequency symptoms and bladder capacity are more readily explained by its concomitant anticholinergic properties.

Amitriptyline also has serotonergic effects by its inhibition of 5-HYDROXYTRYPTAMINE reuptake. This mechanism of action is thought to occur within the spinal and supraspinal neuronal pathways that coordinate bladder function. ${ }^{27}$
Current data indicate that 5-hydroxytryptamine receptor activation, induced by amitriptyline, affects the neuromodulation of afferent and efferent nerve pathways that signal pain and urgency sensation within the bladder. In addition, amitriptyline might improve IC symptoms through its antihistamine properties. Amitriptyline is a potent tricyclic antidepressant in terms of blocking $\mathrm{H}_{1}$-specific receptors, including those on mast cells. Adverse effects associated with amitriptyline's anticholinergic properties occur in over $90 \%$ of patients receiving the drug, and considerably limit its use. ${ }^{9}$ Nonetheless, this tricyclic antidepressant represents a powerful, safe, and apparently effective tool in the treatment of IC pain. A beneficial side effect of this drug is its sedative properties, which can promote restorative sleep. This can vastly improve the quality of life of IC patients, although this sedative effect can also limit the tolerated dose.

\section{Gabapentin}

The use of the anticonvulsant agent gabapentin for IC pain relies on the drug's well-documented efficacy in chronic pain conditions. The mechanism of action of gabapentin is not fully understood, but the agent has been shown to be effective for neuropathic pain in conditions such as diabetic neuropathy, reflex sympathetic dystrophy, and postherpetic neuralgia. ${ }^{28}$ It could be considered an ideal drug, as it does not require monitoring of blood levels, unlike other anticonvulsants such as carbemazapine. Additionally, gabapentin is not metabolized by the liver, but is excreted unchanged in the urine. Over the past decade, the use of gabapentin for IC pain has increased anecdotally, but no clinical trials have tested its efficacy in a randomized, prospective, double-blind setting. Most descriptions of its use in the literature are case reports and open-label studies.

Gabapentin is structurally related to the neurotransmitter gamma-aminobutyric acid (GABA); however, it is not known to bind to GABA receptors or have any effect on the activity or metabolism of GABA. Even less is known about its effect on analgesia, although it is believed to modulate calcium channels, specifically at the $\alpha-2-\gamma$ subunit. ${ }^{29}$ These channels are thought to be specifically associated with neuropathic pain.

There is also evidence that gabapentin might exert some activity against the N-methyl-Daspartate (NMDA) receptor. It is believed that gabapentin competes with other compounds 
at the NMDA receptor, which indicates that it has activity against this receptor. ${ }^{30}$ Gabapentin is thought to exhibit an indirect action on the NMDA receptor, thereby improving pain symptoms, in addition to reducing sympathetic tone in the bladder. Furthermore, it is known that gabapentin enhances endogenous opioid release in the limbic system, subsequently increasing pain control. ${ }^{30}$ This mechanism might also enable patients to reduce their use of exogenous narcotics while achieving the same level of pain control.

A single-center, clinical trial by Sasaki in 2001 reported the efficacy of gabapentin on refractory genitourinary-tract pain. ${ }^{31}$ Pain reduction was observed in nearly $50 \%$ of the 21 patients treated with gabapentin. This encouraging outcome was seen in a patient population with refractory pelvic pain that was very difficult to manage, and oral gabapentin might be more successful in the treatment of newly diagnosed IC pain. Additionally, Hansen reported significant improvements in IC pain in two patients in a case report. ${ }^{32}$ Given its minimal adverse-effect profile and overall safety, gabapentin can be used effectively and safely by physicians treating IC pain. Dosing can begin at $100 \mathrm{mg}$ daily and be titrated up to $3.6 \mathrm{~g}$. Although gabapentin has shown promise as an adjunctive treatment in the management of various chronic pain syndromes, its efficacy in treating IC pain will have to be established in a multicenter, randomized, clinical trial.

Pregabalin is a drug similar to gabapentin, and it is actively being studied as a treatment for neuropathic pain in disease states such as diabetic neuropathy and postherpetic neuralgia. Pregabalin selectively binds to the $\alpha-2-\gamma$ subunit of calcium channels, thereby reducing calcium influx and modulating release of downstream excitatory neurotransmitters. ${ }^{33}$ A randomized, controlled trial designed to treat pain associated with diabetic neuropathy showed a 50\% decrease in pain symptoms. ${ }^{34}$ Such promising results in treating neuropathic pain are encouraging and suggest that pregabalin might have a role in treating IC pain; however, no clinical trials have investigated its use in IC.

\section{Immunosuppressive agents}

Efforts to use immunosuppressive drugs such as prednisone or cyclosporine A (CA) (also known as ciclosporin) have shown some success in treating the pain, and some other symptoms, associated with IC. Immunosuppressive agents are thought to reduce the inflammatory response in the bladder and to treat empirically the autoimmune processes thought to be associated with IC. In a small, Canadian study 14 patients with the ulcerative form of IC were treated with $25 \mathrm{mg}$ of prednisone daily. ${ }^{35} \mathrm{~A}$ decrease in pain symptoms was reported by $69 \%$ of these patients. Although the results of this study are impressive, it was small, and only targeted patients with confirmed Hunner's ulcers. This limits the applicability of prednisone, as approximately $90 \%$ of patients with IC do not have the ulcerative form of the disease. Another factor that could limit the use of immunosuppressants for the treatment of IC is that diabetic control was compromised by the use of steroids in some of the patients in the study. Despite these limiting factors, the study does suggest a potentially new area of therapy for IC pain.

The use of low-dose CA for the treatment of IC has also been investigated by a group in Finland. ${ }^{36,37}$ In their original report, 11 patients with IC were initially treated with $2.5-5.0 \mathrm{mg} / \mathrm{kg}$ of CA daily, which was then reduced to a $1.5-3.0 \mathrm{mg} / \mathrm{kg} /$ day maintenance dose for 3-6 months. Bladder pain either decreased or disappeared in 10 patients, in addition to significant increases in mean and maximum voided volumes and reduction in voiding frequency.

In a more recent study from the same authors, reporting the effects of long-term treatment ( $>1$ year) with CA (initially $3 \mathrm{mg} / \mathrm{kg} /$ day, gradually decreasing to as low as $1 \mathrm{mg} / \mathrm{kg} /$ day) 20 out of 23 patients were completely relieved of their bladder pain. ${ }^{37}$ Furthermore, mean 24-h urinary frequency decreased from 20.8 (standard deviation [SD] 6.3) to 10.2 (SD 3.8), mean maximal bladder capacity increased from $161.8 \mathrm{ml}$ (SD 74.6 ) to $360.7 \mathrm{ml}$ (SD 99.3), and mean voided volume increased from $101.4 \mathrm{ml}$ (SD 42.7) to $246.4 \mathrm{ml}$ (SD 97.9), all of which were statistically significant compared to baseline. It should be noted that symptoms recurred in all nine patients who stopped CA. No renal toxicity occurred; however, seven patients developed adverse effects such as hypertension, gingival hyperplasia, and increased hair growth. There is concern about the development of skin malignancies with long-term use of CA, and one patient developed a basalioma after 5 years of treatment.

While these immunosuppressive drugs, particularly prednisone and CA, have shown 


\section{GLOSSARY}

$T_{H} 1$ CELLS

Cells that participate in cell-mediated immunity and are essential for controlling intracellular pathogens as viruses and certain bacteria

$T_{H} 2$ CELLS

Cells that provide help for $B$ cells and are essential for antibody-mediated immunity

Table 2 Summary of invasive therapy for interstitial cystitis.

\begin{tabular}{lll}
\hline Therapy & Efficacy of treatment & Evidence of efficacy \\
\hline Hydrodistension & $\begin{array}{l}12-70 \% \text { of patients had symptom } \\
\text { improvement }\end{array}$ & $\begin{array}{l}\text { Nonrandomized open-label } \\
\text { studies }^{39,40}\end{array}$ \\
\hline Bacillus Calmette-Guèrin & $\begin{array}{l}\text { No benefit over placebo } \\
\text { Resiniferatoxin }\end{array}$ & $\begin{array}{l}\text { Randomized, controlled trial } \\
\text { No benefit over placebo }\end{array}$ \\
Dimethylsulfoxide & $\begin{array}{l}50-93 \% \text { of patients had improved pain } \\
\text { symptoms (high relapse rate) }\end{array}$ & Randomized, controlled trial \\
\hline
\end{tabular}

some early promise, it is clear that larger, prospective, randomized trials are still needed to demonstrate therapeutic improvement in IC symptoms before their widespread use can be advocated.

\section{INVASIVE TREATMENTS}

Pain management for IC can also involve more invasive treatments such as intravesical instillations and surgical procedures. A detailed discussion involving the surgical management of IC pain is beyond the scope of this paper; however, a brief review of hydrodistension, intravesical agents, and sacral neuromodulation follows (Table 2).

\section{Hydrodistension}

Cystoscopy, combined with hydrodistension, under anesthesia, is often the first diagnostic and therapeutic choice for IC in many urologic practices. If it is considered necessary to conduct a bladder biopsy, it can be performed at the same time. The efficacy of hydrodistension has proven to be variable. ${ }^{38}$ Hanno and Wein have described response rates ranging from $12 \%$ to $26 \% .{ }^{39} \mathrm{~A}$ more recent study from Japan reported a $70 \%$ improvement in symptoms in patients whose bladder capacity is greater than $100 \mathrm{ml}$ following hydrodistension under anesthesia. ${ }^{40}$ The mechanism of action behind this treatment is believed to be a mechanical stretch of the bladder mucosa and damage to the submucosal neuronal plexus, which thereby decreases pain transmission through the afferent fibers. Although treatment efficacy can range from $12-70 \%$, the effects of therapy have been reported to be brief, lasting no longer than 3-6 months. Nonetheless, this therapy can be useful in managing the pain from IC in many circumstances.

\section{Dimethylsulfoxide}

With the exception of PPS, intravesical dimethylsulfoxide (DMSO) is the only other
FDA-approved drug for the treatment of IC. The mechanism of action is not entirely clear, but DMSO is known to deplete substance $\mathrm{P}$ in the bladder and to stimulate mast-cell degranulation. ${ }^{41}$ Originally popularized by Stewart et al. in 1967, who reported overall improvement in IC symptoms (not specifically pain) in up to $75 \%$ of patients, ${ }^{42}$ more recent DMSO trial results have not been encouraging. In a 1998 study, Perez-Marrero et al. showed improvements in pain symptoms in $93 \%$ of patients versus $35 \%$ improvement in the salineplacebo group. Unfortunately, the same study revealed that $59 \%$ of the patients relapsed in the following 4 weeks. ${ }^{43}$ The same authors later showed that the beneficial effects of DMSO instillation can be moderately prolonged with the use of heparin..$^{44}$ In a European study of 28 patients, a modest response to DMSO instillations was also noted. ${ }^{45}$ More importantly, the study showed that the adverse effects associated with DMSO therapy could be well tolerated, so that a 6-week course of treatment could generally be completed. Urethral irritation was evident in nearly $50 \%$ of patients, but was reported as tolerable. Although certainly not a long-lasting treatment for pain and other symptoms associated with IC, DMSO instillation offers another tool in the treatment of IC pain.

\section{Bacillus Calmette-Guèrin and resiniferatoxin}

The use of intravesical bacillus Calmette-Guèrin (BCG) for the treatment of IC is based on the proposed role of immune dysregulation in the etiology of IC, specifically an imbalance of the $\mathrm{T}_{\mathrm{H}} 1$ CELLS and $\mathrm{T}_{\mathrm{H}} 2$ CELLS. First reported in an open-label study by Zeidman et al., in which improvement was seen in all categories of IC symptoms, BCG treatment seemed promising. ${ }^{46}$ Peters et al. performed a small, randomized, placebo-controlled study and reported that $67 \%$ of patients treated with BCG noticed an 
improvement in IC symptoms, compared to 33\% in the placebo group. ${ }^{47}$ With respect to pelvic pain, $53 \%$ of BCG-treated and 33\% of placebotreated patients had a subjective improvement in symptoms. When followed for at least 2 years, $81 \%$ of initial BCG responders continued to experience reduced pelvic pain. ${ }^{48}$ By contrast, a recently published large-scale study conducted by the Interstitial Cystitis Clinical Trials Group demonstrated little benefit for this therapy when compared to placebo; $21 \%$ versus $12 \%$, respectively, reported moderate or marked improvement in their IC symptoms on global response assessment $(P=0.06) .{ }^{49}$

Similarly, resiniferatoxin had theoretical promise as an intravesical agent to help control pain in patients with IC. It is a vanilloid-receptor agonist that desensitizes C-fibers that transmit pain within the bladder. In a randomized, double-blind, placebo-controlled trial published in 2005 , no statistically significant benefit was seen with the use of this agent. ${ }^{50}$ On the basis of currently available evidence, both BCG and resiniferatoxin should still be considered investigational interventions for the management of IC symptoms.

\section{Sacral neuromodulation}

The use of sacral nerve-root stimulation for the treatment of bladder dysfunction has been well described since Schmidt, Bruschini, and Tanagho reported on this technique in 1979.51 Most studies have evaluated the effects of sacral neuromodulation on urinary frequency, urgency, incontinence, and nonobstructive urinary retention. ${ }^{52,53}$ An implantable, neuroprosthetic device that stimulates the transforaminal sacral nerve (InterStim ${ }^{\circledR}$, Medtronic Inc., Minneapolis, $\mathrm{MN}$ ) has been FDA-approved for these symptoms. There is a paucity of information available about the effects of sacral neuromodulation on chronic pain.

Studies have suggested that pelvic pain can be ameliorated in some instances by the use of this device. ${ }^{54,55}$ In a study of just 10 patients, Siegel et al. demonstrated that 9 out of 10 patients received some level of pain relief with sacral-nerve stimulation. ${ }^{54}$ Similarly, a more recent study revealed that nearly two-thirds of IC patients treated with sacral-nerve stimulation had reduced pain following implantation. ${ }^{55}$ Peters et al. reported a $36 \%$ decrease in narcotic use, as defined by morphine dose equivalents, after implantation of the
InterStim $^{\circledast}$ device, with 4 out of 18 patients completely stopping narcotic treatment. ${ }^{56}$ These data are preliminary and involve small numbers of patients, and it is clear that this mode of therapy needs further investigation. Sacral neuromodulation might, however, offer a promising option for the treatment of IC in the future, including managing the pain associated with this disorder.

\section{CONCLUSION}

IC remains a frustrating clinical problem for any physician treating this disorder, particularly with regard to the debilitating pain associated with IC. The pathophysiology of this disease remains poorly understood, but current knowledge has spurred the investigation of novel therapies. A multitude of pain-relieving oral agents are now available to patients with IC, including narcotics, antidepressants, gabapentin, and PPS. While intravesical therapy has not been shown to be particularly successful, some instillations might help to temporarily alleviate symptoms, particularly in the management of chronic pain and acute flares.

There are also a limited number of surgical options available that could potentially treat the pain caused by IC. A multimodal approach is probably necessary to obtain the most successful outcomes. IC remains a painful clinical entity that requires additional investigation to understand its pathophysiology, and further improvements in pain management.

Note added in proof While this article was in press, a prospective, open label, randomized trial has been published comparing the use of CA to PPS in the treatment of IC. ${ }^{57}$ A group of 64 patients were randomized to receive either a low dose of CA $(3 \mathrm{mg} / \mathrm{kg})$ or PPS $100 \mathrm{mg}$ three times a day. CA was superior to PPS in all clinical outcomes. CA treatment at 6-month follow-up showed a $34 \%$ decrease in urinary frequency, while no improvement was seen in the PPS group. Additionally, the clinical response rate as measured by the global response assessment improved in $75 \%$ of the patients in the CA group, compared to only $19 \%$ of those in the PPS group. These promising results continue to suggest that IC symptoms can be successfully managed with low-dose CA therapy. Long-term studies are clearly required, with particular attention paid to the potential adverse effects of CA. 


\section{KEY POINTS}

- Interstitial cystitis (IC) is characterized by lower urinary tract symptoms of frequency, dysuria, urgency and chronic pelvic pain

- The pathophysiology of IC is poorly understood, and several mechanisms have been implicated in this chronic condition

- The majority of treatments for IC are aimed at treating the pain associated with the disorder, which is the most debilitating symptom

- Several oral agents have been shown to be effective in controlling the pain associated with IC in some patients, including narcotics, antidepressants, gabapentin and pentosan polysulfate

- Some invasive therapies such as hydrodistension, intraveslcal treatments and sacral neuromodulation are also being investigated for the treatment of IC

\section{References}

1 Nordling J (2004) Interstitial cystitis: how should we diagnose it and treat it in 2004? Curr Opin Urol 14: 323-327

2 Hanno P et al. (2005) International consultation on IC-Rome, September 2004/Forging an international consensus: progress in painful bladder syndrome/ interstitial cystitis. Int Urogynecol J Pelvic Floor dysfunct 16 (Suppl 1): S2-S34

3 Jones CA and Nyberg L (1997) Epidemiology of interstitial cystitis. Urology 49: 2-9

4 Parsons CL (2003) Prostatitis, interstitial cystitis, chronic pelvic pain, and urethral syndrome share a common pathophysiology: lower urinary dysfunctional epithelium and potassium recycling. Urology 62 : 976-982

5 Foster HE (1999) Urologic causes of pelvic pain. Infertility Reprod Med Clin North Am 10: 701-715

6 Oleary MP et al. (1997) The interstitial cystitis symptom index and problem index. Urology 49 (Suppl 5A): S58-S63

7 Parsons CL et al. (2002) Increased prevalence of interstitial cystitis: previously unrecognized urologic and gynecologic cases identified using a new symptom questionnaire and intravesical potassium sensitivity. Urology 60: 573-578

8 Propert KJ et al. (2002) Pitfalls in the design of clinical trials for interstitial cystitis. Urology 60: 742-748

9 Rovner E et al. (2000) Treatments used in women with interstitial cystitis: the interstitial cystitis data base (ICDB) study experience. The Interstitial Cystitis Data Base Study Group. Urology 56: 940-945

10 Brookoff D (1997) The causes and treatment of pain in interstitial cystitis. In Interstitial Cystitis, 247-255 (Ed Sant GR) Philadelphia: Lippincott-Raven

11 Ratliff TL et al. (1994) The etiology of interstitial cystitis. Urol Clin N Amer 21: 21-30

12 Henry RA et al. (2001) Alkalinized intravesical lidocaine to treat interstitial cystitis: absorption kinetics in normal and interstitial cystitis bladders. Urology 57 (Suppl 1): S119

13 Parsons CL (2005) Successful downregulation of bladder sensory nerves with combination of heparin and alkalinized lidocaine in patients with interstitial cystitis. Urology 65: 45-48

14 Wong D et al. (1998) Nalmefene: a long acting opioid. J Emerg Med 16: 471-475
15 Stone N (1994) Nalmefene in the treatment of interstitial cystitis. Urol Clin North Am 21: 101-106

16 Lynes WL et al. (1987) Mast cell involvement in interstitial cystitis J Urol 138: 746-752

17 Theoharides TC et al. (1997) Hydroxyzine therapy for interstitial cystitis. Urology 49 (Suppl 5A): S108-S110

18 Sant GR et al. (2003) A pilot clinical trial of oral pentosan polysulfate and oral hydroxyzine in patients with interstitial cystitis. J Urol 170: 810-815

19 Casale TB et al. (1984) Induction of human cutaneous mast cell degranulation by opiates and endogenous opioid peptides: evidence for opiate and nonopiate receptor participation. J Allergy Clin Immunol 73: 775-781

20 Sant GR and Theoharides TC (1994) The role of the mast cell in interstitial cystitis. Urol Clin North Am 21: 41-53

21 Minogiannis P et al. (1998) Hydroxyzine inhibits neurogenic bladder mast cell activation. Int $J$ Immunopharmacol 20: 553-563

22 Kahn B et al. (2005) Management of patients with interstitial cystitis or chronic pelvic pain of bladder origin: a consensus report. Curr Med Res Opin 21: 509-516

23 Mulholland SG et al. (1990) Pentosan polysulfate sodium for the therapy of interstitial cystitis-a double blind placebo controlled clinical study. Urology 35: 552-558

24 Nickel JC et al. (2005) Randomized, double-blind, dose-ranging study of pentosan polysulfate sodium for interstitial cystitis. Urology 65: 654-658

25 Hanno PM and Wein AJ (1987) Medical treatment of interstitial cystitis (other than Rimso-50/Elmiron). Urology 29 (Suppl): S22-S26

26 van Ophoven A et al. (2004) A prospective, randomized, placebo controlled, double-blind study of amitriptyline for the treatment of interstitial cystitis. J Urol 172: 533-536

27 Pilowsky let al. (1982) A controlled study of amitriptyline in the treatment of chronic pain. Pain 14: 169-179

28 Backonga M and Glanzman RL (2003) Gabapentin dosing for neuropathic pain: evidence from randomized, placebo-controlled clinical trials. Clin Ther 25: 81-104

29 Gee NS et al. (1996) The novel anticonvulsant drug, gabapentin (Neurontin), binds to the alpha2delta subunit of a calcium channel. J Biol Chem 271: 5768-5776

30 Nicholson B (2000) Gabapentin use in neuropathic pain syndromes. Acta Neurol Scand 101: 359-371

31 Sasaki K et al. (2001) Oral gabapentin treatment for refractory genitourinary tract pain. Tech Urol 7: 47-49

32 Hansen $\mathrm{H}$ (2000) Interstitial cystitis and the potential role of gabapentin. South Med J 93: 238-242

33 Hindmarch I et al. (2005) A double-blind, placebo- and positive-internal-controlled (alprazolam) investigation of the cognitive and psychomotor profile of pregabalin in healthy volunteers. Psychopharmacology 183: 133-143

34 Freyhagen $\mathrm{R}$ et al. (2005) Efficacy of pregabalin in neuropathic pain evaluated in a 12-week, randomised, double-blind, multicentre, placebocontrolled trial of flexible- and fixed-dose regimens. Pain 115: 254-263

35 Soucy F and Gregoire M (2005) Efficacy of prednisone for severe refractory ulcerative interstitial cystitis. J Urol 173: 841-843

36 Forsell T et al. (1996) Cyclosporine in severe interstitial cystitis. J Urol 155: 1591-1593 
37 Sairanen J et al. (2004) Long-term outcome of patients with interstitial cystitis treated with low dose cyclosporine A. J Urol 171: 2138-2141

38 Turner K and Stewart H (2005) How do you stretch a bladder? A survey of UK practice, literature review, and a recommendation of a standard approach. Neurourol Urodyn 24: 74-76

39 Hanno P and Wein A (1991) Conservative therapy for interstitial cystitis. Semin Urol 9: 143-147

40 Yamada Tet al. (2003) Adjuvant hydrodistension under epidural anesthesia for interstitial cystitis. Int J Urol 10: 463-468

41 Moldwin R and Sant GR (2002) Interstitial cystitis: a pathophysiology and treatment update. Clin Obstet Gynecol 45: 259-272

42 Stewart BH et al. (1967) The use of dimethyl sulfoxide (DMSO) in the treatment of interstitial cystitis. J Urol 98: 671-672

43 Perez-Marrero R et al. (1988) A controlled study of dimethyl sulfoxide in interstitial cystitis. J Urol 140: 36-39

44 Perez-Marrero R et al. (2003) Prolongation of response to DMSO by heparin maintenance. Urology 41: 64-66

45 Rossberger J et al. (2005) Critical appraisal of dimethyl sulfoxide treatment for interstitial cystitis: discomfort, side effects and treatment outcome. Scand J Urol Nephrol 39: 73-77

46 Zeidman EJ et al. (1994) Bacillus Calmette-Guerin immunotherapy for refractory interstitial cystitis. Urology 43: 121-124

47 Peters D et al. (1997) The efficacy of intravesical Tice strain bacillus Calmette-Guèrin in the treatment of interstitial cystitis: a double-blind, prospective, placebo controlled trial. J Urol 157: 2090-2094
48 Peters KM et al. (1998) The efficacy of intravesical bacillus Calmette-Guerin in the treatment of interstitial cystitis: long term followup. J Urol 159: 1483-1486

49 Mayer R et al. (2005) A randomized controlled trial of intravesical bacillus Calmette-Guèrin for treatment refractory interstitial cystitis. J Urol 173: 1186-1191

50 Payne CK et al. (2005) Intravesical resiniferatoxin for the treatment of interstitial cystitis: a randomized, double blind, placebo controlled trial. J Urol 173: 1590-1594

51 Schmidt RA et al. (1979) Urinary bladder and sphincter responses to stimulation of dorsal and ventral sacral roots. Invest Urol 16: 300-304

52 Hassouna MM et al. (2000) Sacral neuromodulation in the treatment of urgency-frequency symptoms: a multicenter study of efficacy and safety. J Urol 163: 1849-1854

53 Abrams P et al. (2003) The role of neuromodulation in the management of urinary urge incontinence. BJU Int 91: 355-359

54 Siegel S et al. (2001) Sacral nerve stimulation in patients with intractable pelvic pain. J Urol 166: 1742-1745

55 Peters KM and Carey JM (2003) Sacral neuromodulation for the treatment of refractory interstitial cystitis: outcomes based on technique. Int Urogynecol J Pelvic Floor Dysfunct 14: 223-228

56 Peters KM and Konstandt D (2004) Sacral neuromodulation decreases narcotic requirements in refractory interstitial cystitis. BJU Int 93: 777-779

57 Sairanen J et al. (2005) Cyclosporin A and pentosan polysulfate sodium for the treatment of interstitial cystitis. J Urol 174: 2235-2238

\section{Competing interests}

HE Foster Jr declared competing interests; go to the article online for details. S Phatak declared he has no competing interests. 